\title{
Atherectomy Cutter Diameter
}

National Cancer Institute

\section{Source}

National Cancer Institute. Atherectomy Cutter Diameter. NCI Thesaurus. Code C150233.

The diameter of the cutter component for atherectomy devices that is used to cut the plaque/thrombus. 